\title{
ESTUDIO DEL FORAMEN VENOSO ESFENOIDAL EN POBLACIÓN CHILENA MEDIANTE MÉTODOS IMAGENOLÓGICOS TRIDIMENSIONALES
}

\author{
Andrés Melián ${ }^{1-2}$, María F. Cortés-Sylvester ${ }^{1-3}$, Paulette Palyee ${ }^{1}$, Camila Boin ${ }^{4}$ \\ ${ }^{1}$ Departamento de Anatomía, Facultad de Odontología, Universidad Finis Terrae, Santiago, \\ Chile \\ ${ }^{2}$ Servicio de Cirugía Maxilofacial, Hospital San Juan de Dios, Santiago, Chile. \\ ${ }^{3}$ Departamento de Patología y Medicina Oral, Área de Radiología, Facultad de Odontología, \\ Universidad de Chile, Santiago, Chile \\ ${ }^{4}$ Facultad de Odontología, Universidad de Chile, Santiago, Chile
}

\section{RESUMEN}

Introducción: El foramen venoso es un reparo anatómico inconstante localizado en la base de cráneo, específicamente en el ala mayor del esfenoides, anteromedial al foramen oval. Éste permite el paso de una vena emisaria esfenoidal, la cual conecta al plexo pterigoideo con el seno cavernoso. Su presencia se ha relacionado con complicaciones clínicas en procedimientos neuroquirúrgicos y es una potencial vía de acceso de procesos infecciosos a la cavidad craneal. Objetivo: Determinar la prevalencia y las características morfológicas más prevalentes del foramen venoso analizadas mediante tomografía computarizada de haz cónico (CBCT). Material y método: Se estudiaron 126 CBCT de adultos chilenos disponibles en el departamento de anatomía de la Universidad Finis Terrae, en un análisis estadístico donde se observaron las variaciones en la incidencia, morfología, permeabilidad y distancia a otras estructuras anatómicas: foramen espinoso, foramen oval y línea media. Resultados: Se observó la presencia del foramen Venoso en un 19\% de la población. $87.5 \%$ se encontró unilateralmente y $12.5 \%$ bilateralmente. El $48,1 \%$ fueron redondeados y el $51,9 \%$ irregulares. El diámetro promedio fue de $2.2 \mathrm{~mm}$, con un $100 \%$ de ellos permeables. Las distancias promedio entre el foramen Venoso y el foramen oval, el foramen espinoso y la línea media fueron $1.72 \mathrm{~mm}, 10.14 \mathrm{~mm}$ y $19.7 \mathrm{~mm}$ respectivamente. Conclusiones: El foramen venoso se presentó en el $19 \%$ del total, en forma ovalada o irregular, anteromedial al foramen oval, presentándose principalmente de manera unilateral. Dichas características anatómicas de este foramen deben considerarse durante las intervenciones neuroquirúrgicas en la fosa craneal media.

Palabras clave: hueso esfenoides; tomografía computarizada de haz cónico; foramen de Vesalius; vena emisaria.

\begin{abstract}
Introduction: The foramen venosum is an inconstant anatomical hole located at the cranial base, specifically at the greater wing of the sphenoid, anterior medial to the foramen ovale. It allows the passage of a sphenoid emissary vein, which connects the pterygoid plexus with the cavernous sinus. Its presence has been related to clinical complications in neurosurgical procedures and is a potential access route to infectious processes in the cranial cavity. Objective: Determine the prevalence and morphological characteristics of the foramen venosum analyzed by means of cone beam computed tomography (CBCT). Material and method: 126 CBCT of Chilean adults available in the Department of Anatomy of the Finis Terrae University were studied, in a statistical analysis where variations in the incidence, morphology, permeability and distance to other anatomical structures were observed: foramen spinosum, foramen ovale and midline. Results: The presence of the foramen venosum was observed in $19 \%$ of the population. $87.5 \%$ were found unilaterally and $12.5 \%$ bilaterally. $48.1 \%$ were rounded and $51.9 \%$ irregular. The average diameter was $2.2 \mathrm{~mm}$, with $100 \%$ of them permeable. The average distances between the foramen venosum and the foramen ovale, the foramen spinosum and the midline were $1.72 \mathrm{~mm}$, $10.14 \mathrm{~mm}$ and $19.7 \mathrm{~mm}$. respectively. Conclusions: The foramen venosum appeared in $19 \%$ of the total, in an oval or irregular shape, anterior and medial to the foramen ovale, presenting mainly unilaterally. These anatomical characteristics of this foramen must be considered during neurosurgical interventions in the middle cranial fossa.
\end{abstract}

Key words: Sphenoid bone; cone beam computed tomography; Vesalius foramen; emissary vein.

* Correspondencia a: Andrés Melián.
andresmelianrivas@yahoo.es

Recibido: 30 de Marzo, 2020. Revisado: 15 de Abril, 2020. Aceptado: 20 de Abril, 2020. 


\section{INTRODUCCIÓN}

En la concavidad interna de las alas mayores del hueso esfenoides se encuentra un foramen inconstante, el foramen venoso (FV), conocido antiguamente como foramen de Vesalio (Rouviere y Delmas, 2005). Éste, es un foramen que no se ha descrito en ningún otro primate, siendo un rasgo distintivo de los humanos (Berge y Bergman, 2001); localizado en la porción cóncava del ala mayor del Esfenoides en la fosa craneal media, antero-medial al foramen oval (FO), al foramen espinoso (FE), al canal carotídeo y posteromedial al foramen redondo (FR) (Rouviere y Delmas, 2005) (Fig. 1). Su origen se relacionaría con el $\mathrm{FO}$, ya que se formaría producto de la osificación de una espícula ósea que atravesaría al FO dando como resultado dos forámenes con características anatómicas propias, siendo uno de los motivos de la estrecha relación de ambos reparos anatómicos (Bayrak et al. 2018).

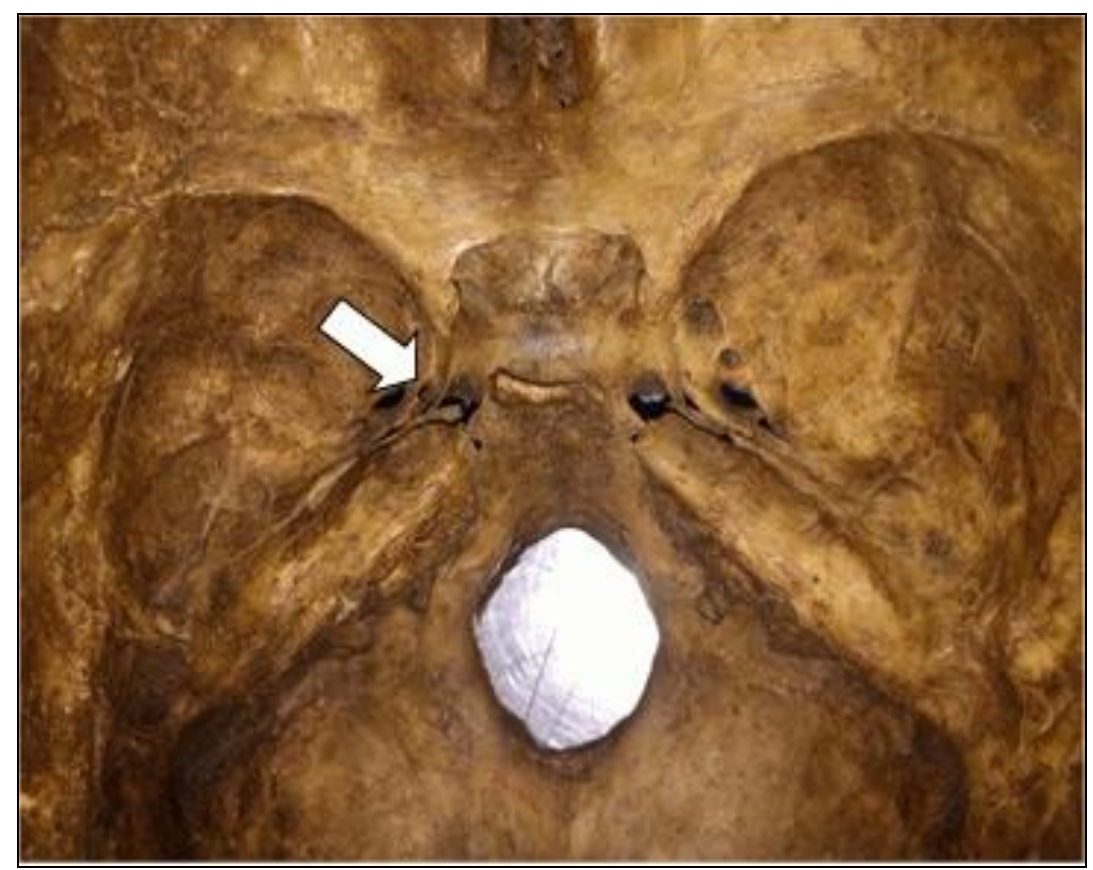

Figura 1: Vista endocraneal base de cráneo. Foramen venoso señalado con flecha blanca.

La presencia de este foramen permite el paso de una vena emisaria esfenoidal la cual comunica el plexo pterigoideo y el seno cavernoso, siendo una vía de comunicación entre el drenaje venoso craneal interno y externo (Lanzieri et al. 1988; Gupta et al. 2005; Dogan et al. 2014), incluso se ha reportado que puede transmitir a la arteria meníngea accesoria (Kale et al. 2009). Esta comunicación pudiese ser motivo de complicaciones clínicas, tales como el paso de procesos infecciosos desde exocráneo al seno cavernoso, asociado a complicaciones como trombosis séptica de seno cavernoso, meningitis bacteriana, abscesos e infartos cerebrovasculares (Freire et al. 2013). También se han reportado complicaciones clínicas asociadas a técnicas neuroquirúrgicas de tratamiento de neuralgia del Nervio trigémino, existiendo terapias que involucran abordajes quirúrgicos que utilizan al $\mathrm{AO}$ como vía de acceso al ganglio trigeminal (Ozer y Govsa, 2014), pudiendo la proximidad y presencia del FV al FO provocar complicaciones potencialmente graves (Rossi et al. 2010; Natsis et al. 2018). Los diversos estudios realizados para investigar morfología de estructuras anatómicas se han centrado principalmente en el estudio de osamentas, mediante visión y medición directa (Ukoha et al. 2018); en algunos casos los estudios se complementaron con estudio fotográfico y medición computacional para obtener medidas más precisas (Chaisuksunt et al. 2012). Otro método de utilidad, pero menos usado, es el método imagenológico tridimensional; donde se han reportado análisis de forámenes de base craneal mediante tomografía axial computarizada (Gingsberg et al. 1994; Ginat et al. 2013). Sin embargo, pocos estudios han utilizado la 
tecnología CBCT para este tipo de análisis (Bayrak et al. 2018; Görürgöz y Paksoy, 2020) siendo una alternativa ventajosa en el análisis del FV.

El objetivo de este estudio es determinar la presencia y las características morfológicas más frecuentes del FV analizados en una población chilena mediante estudios imagenológicos tridimensionales.

\section{MATERIAL Y MÉTODO}

Se realizó un estudio descriptivo observacional. Se recolectó una muestra desde la base de datos de imágenes digitales existente en el servicio de imagenología maxilofacial de la Facultad de Odontología de la Universidad Finis Terrae en todos los pacientes sometidos a exámenes CBCT de cráneo completo entre los años 2014 - 2018.

Se incluyeron aquellos volúmenes de CBCT de cráneo completo, que permitiera la visualización de la fosa craneal media al menos desde posterior y lateral al Foramen redondo hasta posterior al FE y lateral al FO del Esfenoides bilateralmente, permitiendo visualización de FV, de existir. Se excluyeron todos aquellos exámenes de pacientes que presentaron anomalías cráneo-faciales, evidencia imagenológica de enfermedades degenerativas óseas o trastornos óseos congénitos, señales de cirugías cráneo-faciales (placas de osteosíntesis, fijaciones rígidas), presencia de lesiones tumorales o neoplásicas de tejidos duros y exámenes con cualquier tipo de artefacto que afectase la zona de interés en grado que determine una calidad de la imagen insuficiente como para evaluar las estructuras anatómicas de estudio.

Para la obtención de dichas imágenes se utilizó el equipo Galileos CBCT Plus con FaceScan (Sirona Systemsinc, Alemania). Estos exámenes fueron indicados previamente en forma justificada por motivos diagnósticos para los pacientes. El campo de visualización (FOV) de dichos exámenes es de $15 \times 15 \times 15 \mathrm{~cm}$ en un volumen esférico. Los exámenes fueron analizados mediante los programas Sidexis XG y Galaxy asociados a la tecnología Dentsply Sirona por un examinador previamente calibrado (test Kappa).

Todos los volúmenes fueron reorientados de tal forma que el plano de Frankfurt fuese paralelo al plano horizontal y los otros dos planos ortogonales perpendiculares a éste. Para cada uno de los volúmenes se recolectaron los siguientes datos: Sexo del paciente, presencia o ausencia de FV, bilateralidad o unilateralidad de éste, forma (ovalada o irregular), presencia 0 ausencia de confluencia de forámenes, diámetro, distancia en milímetros al FO, FE y línea mediana (LM). La medición del diámetro del FV se realizó entre corticales internas de este foramen en su eje mayor; mientras que las distancias entre FV - FO, FV - FE, FV - LM se realizaron desde la cortical interna de cada foramen al otro. El análisis de datos se realizó mediante el software SPSS 10 (SPSS Inc). El valor de nivel de significancia se estableció en $0.05(\alpha=0.05)$. La prueba de T-student para variables independientes se realizó para comparar las medias entre las distintas mediciones obtenidas por lado y género.

\begin{tabular}{|c|c|c|c|c|}
\hline \multirow{2}{*}{ Sexo } & \multicolumn{3}{|c|}{ Presencia (\%) } & \multirow{2}{*}{ Total } \\
\cline { 2 - 4 } & Unilateral & Bilateral & Ausente & $43(34,1 \%)$ \\
\hline Masculino & $7(5,5 \%)$ & $1(0,8 \%)$ & $35(27,8 \%)$ & $43 \%$ \\
Femenino & $14(11,2 \%)$ & $2(1,6 \%)$ & $67(53,1 \%)$ & $83(65,9 \%)$ \\
Total & $21(16,7 \%)$ & $3(2,4 \%)$ & $102(80,9 \%)$ & $126(100 \%)$ \\
\hline
\end{tabular}

Tabla 1: Presencia y distribución de foramen venoso según sexo.

\section{RESULTADOS}

La calibración realizada entre operador y examinador experto para el análisis arrojó en una, una fuerza de concordancia muy buena $(K=0,83)$. De 130 volúmenes del universo de tomografías de cráneo completo realizadas en el servicio de imagenología de la Facultad de
Odontología de la Universidad Finis Terrae que cumplieron con los criterios de inclusión, 126 fueron analizados luego aplicar los criterios de exclusión.

Analizamos 126 volúmenes de los cuales 43 $(34,1 \%)$ eran pacientes del sexo masculino y 83 $(65,9 \%)$ femenino. El FV se encontró presente en $24(19,1 \%)$ volúmenes y ausente en 102 
(80,9\%). En aquellos casos donde se evidenció FV presente, $8(33,3 \%)$ eran de sexo masculino y $16(66,7 \%)$ femenino (Ver tabla 1$).$

De aquellos volúmenes con presencia de $\mathrm{FV}, 3$ $(12,5 \%)$ presentaban FV bilateralmente, mientras que $21(87,5 \%)$ unilateralmente. Dentro de estos últimos, $10(47,6 \%)$ forámenes fueron derechos y $11(52,4 \%)$ izquierdos. Con respecto a la forma, $13(48,1 \%)$ eran de forma redondeada, mientras que $14(51,9 \%)$ de forma irregular, donde del total de forámenes localizados en el lado derecho, $4(30,8 \%)$ eran de forma redondeada y $9(69,2 \%)$ de forma irregular. Mientras que en el lado izquierdo 9 (64,3\%) correspondían a forámenes de forma redondeada y 5 (35,7\%) eran de forma irregular.
El diámetro promedio de los FV analizados fue de $2,27 \mathrm{~mm}$. La media del diámetro del AV en el lado derecho fue de 2,32+/- 0,89 mm con un mínimo de $1,13 \mathrm{~mm}$ y un máximo de $4,21 \mathrm{~mm}$, mientras que en el lado izquierdo la media obtenida fue de $2,11+/-0,36 \mathrm{~mm}$, con una medida mínima de $1,60 \mathrm{~mm}$ y un máximo de 2,85 $\mathrm{mm}$. En adición a esto, $14(59,3 \%)$ de los forámenes encontrados presentaron un diámetro sobre los $2 \mathrm{~mm}$, mientras que $10 \quad(40,7 \%)$ presentó diámetros entre 1 y $2 \mathrm{~mm}$, no se evidenciaron forámenes venosos con medidas inferiores a $1 \mathrm{~mm}$. Se realizaron mediciones desde el FV a otros reparos anatómicos de base de cráneo: FO, FE y LM a cada lado respectivamente (Ver tabla 2).

\begin{tabular}{|lcccc|}
\hline & Mínimo & Máximo & Media & Desv. típ. \\
Diámetro lado derecho & 1,13 & 4,21 & 2,32 & 0,89 \\
Diámetro lado izquierdo & 1,60 & 2,85 & 2,11 & 0,36 \\
Distancia FV-FO (Derecha) & 0,68 & 3,90 & 1,70 & 0,95 \\
Distancia FV-FE (Derecha) & 8,27 & 12,97 & 10,48 & 1,33 \\
Distancia FV-LM (Derecha) & 18,05 & 25,77 & 20,76 & 2,22 \\
Distancia FV-FO (Izquierda) & 0,41 & 4,20 & 1,74 & 1,03 \\
Distancia FV-FE (Izquierda) & 7,37 & 12,43 & 9,82 & 1,35 \\
Distancia FV-LM (Izquierda) & 15,06 & 24,21 & 18,82 & 2,19 \\
\hline
\end{tabular}

Tabla 2: Análisis descriptivo de mediciones obtenidas en milímetros. FV: Foramen venoso; FO: Foramen oval; FE: Foramen espinoso; LM: Línea mediana.

La distancia promedio obtenida desde el FV al FO corresponde a $1,72 \mathrm{~mm}$; la media obtenida en el lado derecho fue de $1,70+/-0,95 \mathrm{~mm}$, mientras que en el lado izquierdo fue de $1,74+/-$ $1,03 \mathrm{~mm}$. Por otra parte, la distancia promedio analizada entre el FV y el FE fue de $10,14 \mathrm{~mm}$, la media obtenida en el lado derecho fue de 10,48 $+/-1,33 \mathrm{~mm}$ y en el lado izquierdo fue de 9,82 +/$1,35 \mathrm{~mm}$. Finalmente la distancia promedio existente entre el FV y la línea mediana en nuestro estudio fue de $19,77 \mathrm{~mm}$ en total, siendo la media en el lado derecho de 20,76 +/- 2,22 $\mathrm{mm}$ y en el lado izquierdo de 18,82 +/- 2,19 mm. Del total de forámenes venosos analizados, sólo se encontró $1(3,7 \%)$ categorizado como una variación anatómica del $\mathrm{FV}$, en la cual se encontró esta estructura anatómica en una posición anterolateral al FO.

No se encontró significación estadística con respecto a la presencia de este reparo anatómico con respecto al sexo de los pacientes. $(p>0,05)$. Cuando la media del diámetro se comparó en base al sexo de los pacientes analizados, no se encontró una diferencia estadísticamente significativa entre los resultados obtenidos ( $p$
$=0,06)$. Tampoco se encontró significancia estadística entre los diámetros obtenidos de los lados derecho e izquierdo $(p=0,432)$. El mismo análisis fue realizado con respecto a las distancias a los diferentes forámenes analizados, donde no se encontró asociación estadística entre el lado y las distancias a los FO $(p=0,932)$ y FE $(p=0,215)$. Sin embargo, esto difirió con respecto a la $L M$, dado que se encontró una diferencia significativamente estadística con respecto a la distancia del FV hasta la línea mediana $(p=0,032)$.

\section{DISCUSIÓN}

La literatura no llega a consenso sobre la presencia de este foramen. En la población estudiada, éste fue del 19\%, independiente del lado y del género siendo similares a los resultados obtenidos por do Nascimento et al. (2018) y Reymond et al. (2005). Sin embargo, hay estudios con porcentajes casi desestimables 
con un 5\% (Srimani et al. 2014) y otros que han descrito un $100 \%$ de presencia, no obstante, este último reporte solo hubo 10 cráneos en estudio (Kaplan et al., 2007).

Los reportes son variados con respecto a la presencia de FV de forma bilateral. En esta población fue de 2,4\%, similar a los estudios realizados por Reymond et al. (2005) y do Nascimento et al. (2018), quienes obtuvieron sólo un $5 \%$ y $6,18 \%$ de incidencia de $\mathrm{FV}$ de forma bilateral. Sin embargo, existen estudios donde se observó esta característica en un $25,1 \%$ y $75 \%$ (Kale et al. 2009; Kodama et al. 1997). En este estudio el hallazgo de forma unilateral del FV (21\%) fue más frecuente que bilateral, resultado similar a los estudios realizados por Boyd (1930), aunque en la mayoría de las investigaciones, el FV se presenta preferentemente de forma unilateral sin mucha variación significativa entre el lado derecho y el lado izquierdo (Figuras 2 y 3 ).

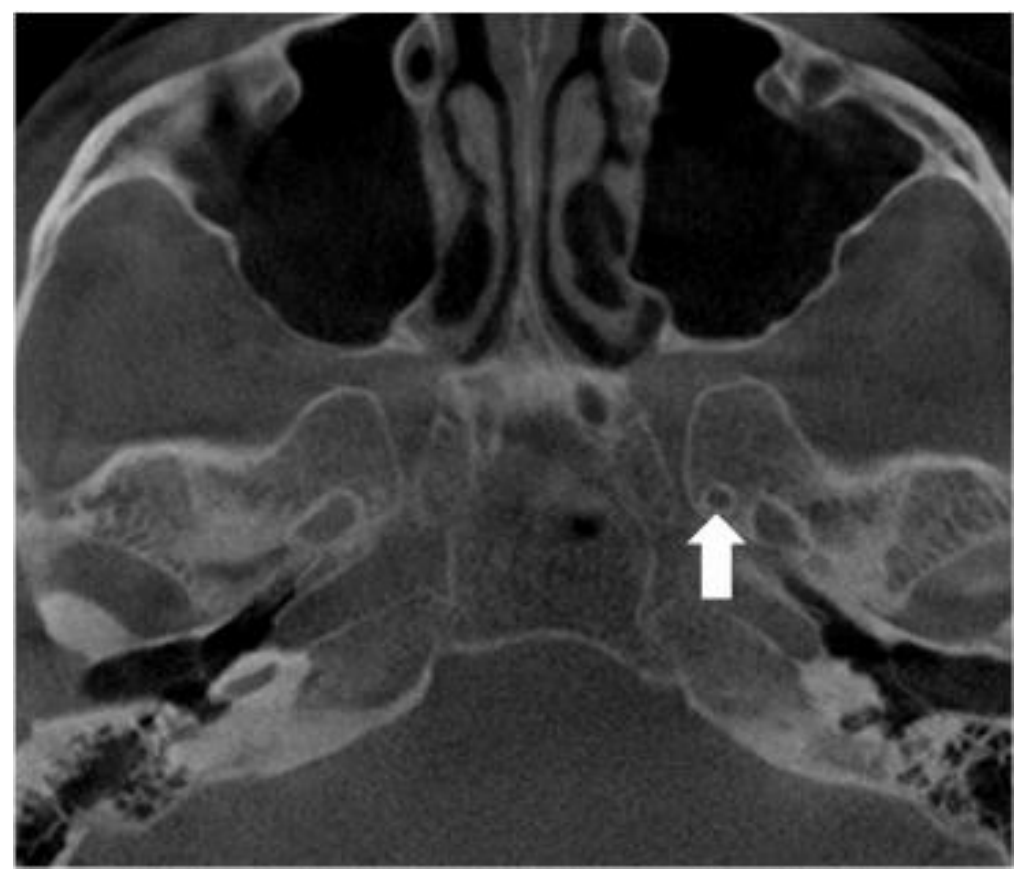

Figura 2: Corte axial de tomografía computarizada de haz cónico. Se observa el foramen venoso señalado con flecha blanca

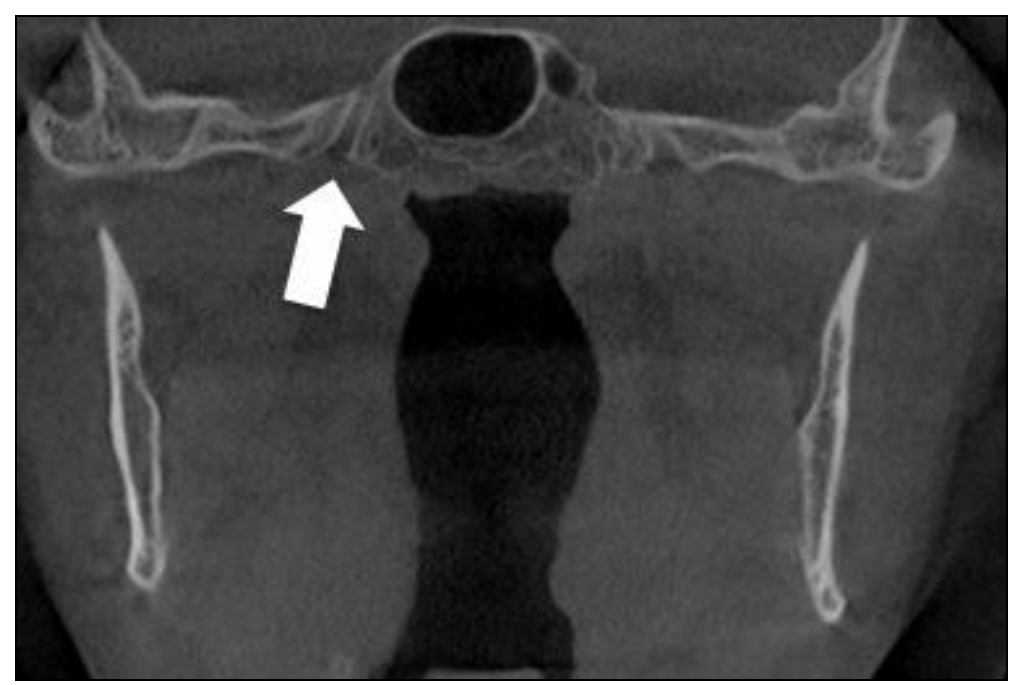

Figura 3: Corte coronal de tomografía computarizada de haz cónico. Se observa el foramen venoso señalado con flecha blanca. 
Con respecto al sexo, los resultados de nuestro estudio concordaron con los resultados obtenidos por Gupta et al. (2005) quien en su investigación con estudio de 35 cráneos obtuvo una mayor presencia de forámenes venosos en mujeres que hombres, tal como nuestro estudio donde de 24 volúmenes donde se observó $\mathrm{FV}$, 16 de estos correspondían a pacientes de sexo femenino y 8 masculino, aunque no se encontró diferencia estadística. Sin embargo, otros resultados son muy variados, en el estudio realizado por Kodama et al. (1997), el 21,5\% de cráneos analizados fueron hombres y $22,8 \%$ fueron mujeres, no encontrándose diferencias significativas entre ambos sexos. Similar al estudio de Chaisuksunt et al. (2012) donde se analizaron 377 cráneos, de los cráneos pertenecientes al sexo masculino $27,2 \%$ presentaron FV mientras que aquellos pertenecientes al sexo femenino 23,3\% presentaron este reparo anatómico. Además del total de mujeres analizadas en nuestro estudio, solo en el 19,2\% de ellas se observó la presencia del FV, mientras que de los hombres analizados un 18,6\% presentó este foramen.

Según Boyd (1930), el 65\% de los FV presentan un diámetro de $0.5-1 \mathrm{~mm}$, en un $5 \%$ el diámetro es de $1 \mathrm{a} 2 \mathrm{~mm}$ y ocasionalmente mide cerca de $2.5 \mathrm{~mm}$. Estos hallazgos no concuerdan con lo observado en nuestro trabajo, donde no se evidenció ningún foramen con diámetro menor a $1 \mathrm{~mm}$. Lazarus et al. (2015) afirmó que un diámetro hasta $0,5 \mathrm{~mm}$ permitiría realizar técnicas quirúrgicas percutáneas de forma segura; sin embargo, nuestro estudio arrojó que la media de diámetro de los forámenes analizados fue de $2,27 \mathrm{~mm}$, muy similar a los resultados obtenidos por do Nascimento et al. (2018) y Bayrak et al. (2018) en este último las medias de los diámetros fluctuaron entre 2,68 $\mathrm{mm}$ y $2,88 \mathrm{~mm}$ siendo esto muy superior a otros estudios como Chaisuksunt et al. (2012) y Shinohara et al. (2010), quienes informaron diámetros de este foramen entre $0,65 \mathrm{~mm}$ y 1,93 $\mathrm{mm}$.

Con respecto a la forma $48,1 \%$ eran redondeados, mientras que $51,9 \%$ fueron irregulares. Estos resultados contrastan con lo publicado en la literatura, dado que la mayoría de los estudios revela que la forma redondeada es mucho más frecuente que la irregular; Raval et al. (2015) encontró forámenes venosos de forma oval en el $96 \%$ e irregulares en el $4 \%$ del total de forámenes analizados.

La distancia promedio existente entre FV y el FO obtenida en nuestro estudio fueron similares a los obtenidos por Gupta et al. (2005), el cual obtuvo un promedio en la distancia de 1,36 mm en el lado derecho y $1,48 \mathrm{~mm}$ en el lado izquierdo. Sin embargo, al igual que los demás parámetros no han sido concordantes, donde incluso se ha reportado distancia promedio de 4,42 mm (Lazarus et al. 2015) siendo casi el doble de lo observado en nuestro estudio. A pesar de que los estudios no tienen valores similares, es importante tener una noción de la proximidad de ambos forámenes. Lanzieri et al. (1998) señaló que el riesgo punción aumenta cuando el FO es mayor de $0,5 \mathrm{~mm}$, por lo tanto, mientras más proximidad de ambas estructuras anatómicas y más grande sea el diámetro de ambos forámenes, más riesgo de complicaciones clínicas. De la misma forma el promedio de la distancia obtenida desde del FV hasta el FE en este estudio fue de $10,48 \mathrm{~mm}$ en el lado derecho y $9,8 \mathrm{~mm}$ en el lado izquierdo; medidas muy similares a aquellos estudios donde se realizó análisis de distancia entre estos dos forámenes como Shinohara et al. (2010) y Ozer y Govsa (2014). Finalmente, las distancias promedio existentes en nuestro estudio entre el FV y la LM fueron diferentes a las evidenciadas en la literatura. Bayrak et al. (2018) obtuvo 19,57 mm en el lado derecho y $15,8 \mathrm{~mm}$ en el lado izquierdo. Lang et al. (1983) $15,78 \mathrm{~mm}$ y 16,72 $\mathrm{mm}$ respectivamente, mientras que Aviles Solis et al. (2011) obtuvo 17, $55 \mathrm{~mm}$ en el lado derecho y $17,95 \mathrm{~mm}$ en el lado izquierdo.

En conclusión, la presencia del FV analizada mediante $\mathrm{CBCT}$ en volúmenes de cráneo completo perteneciente a la base de datos del servicio de imagenología de la Universidad Finis Terrae, fue de un $19 \%$ y se logró observar como una estructura permeable de forma ovalada 0 irregular de 2,2 $\mathrm{mm}$ de diámetro promedio, localizada en una ubicación anteromedial al FO a una distancia promedio de $1,7 \mathrm{~mm}$, presentándose unilateralmente levemente más frecuente en el lado izquierdo, pudiendo manifestarse en menor cantidad de forma bilateral. Por otra parte, se localiza en promedio a $10 \mathrm{~mm}$ del FE y a $19 \mathrm{~mm}$ de la LM. A pesar de estos resultados, la evidencia científica sobre el FV no llega a consenso, debido a la heterogeneidad de la información existente y la falta de uniformidad en los estudios que se han realizado. Aunque existen variados estudios que analizan la morfometría y morfología del FV, la mayoría se ha realizado principalmente en osamentas cadavéricas, mientras que pocos estudios han sido realizados mediante análisis imagenológicos. Este estudio entrega información anatómica detallada sobre esta área en particular, que no ha sido previamente analizada en la población chilena. 


\section{Conflictos de interés.}

Los autores declaran no tener conflictos de interés.

\section{Financiamiento.}

Los autores declaran no haber recibido ningún tipo de financiación económica.

\section{Aprobación ética.}

Este proyecto fue aprobado por el comité de ética de la Universidad FinisTerrae.

\section{Consentimiento informado.}

El departamento de anatomía de la Universidad FinisTerrae cuenta con dispensa de consentimiento informado autorizado por el comité de ética de la Universidad, dado que no se utilizaron datos sensibles de los pacientes y no se publicó información personal de ellos (Resolución №30/2018)

\section{Contribuciones.}

A.M: Recolección, medición de muestras, confección y revisión de manuscrito. MFC: Recolección de muestras, confección y revisión del manuscrito. PP: Recolección de muestra y revisión del manuscrito. CB: Revisión del manuscrito y traducción.

\section{BIBLIOGRAFÍA}

Aviles Solis JC, Olivera Barrios A, De La Garza $C O$, Elizondo Omaña RE, Guzmán-López S. 2011. Prevalencia y características morfométricas del foramen venoso en cráneos del noreste de México. Int J Morphol 29: 158-63.

Bayrak S, Kursun-Cakmak E, Atakan C, Orhan K. 2018. Anatomic Study on Sphenoidal Emissary Foramen by Using Cone Beam computed tomography. The Journal of Craniofacial Surgery 00: 1-3.

Berge JK, Bergman RA. 2001. Variations in size and in symmetry of foramina of the human skull. Clinical Anatomy 4: 406-13.

Boyd GI. 1930. The emissary foramina of the cranium in man and the anthropoids. J Anat 65: 108-21.

Chaisuksunt V, Kwathai L, Namonta K, Rungruang T, Apinhasmit W, Chompoopong $S$. 2012. Occurrence of the foramen of Vesalius and its morphometry relevant to clinical consideration. Scientific World Journal 817454.

Dogan N, Fazhogullari Z, Uysal L, Seker M, Karabulut A. 2014. Anatomical examination of the foramens of the middle cranial fossa. Int. J. Morphol 32: 43-48. do Nascimento JJC, de Silva Neto EJ, de Sliveira Ribeiro EC, de Almeida Holanda MM, Morais Valença M, Oliveira Gomes LD, Alves N. 2018. Foramen venosum in macerated skulls from the North- East of Brazil: morphometric study. Eur J Anat 22: 17-22.

Freire $A R$, Rossi $A C$, de Oliveria VC, Prado FB, Caria PH, Botacin PR. 2013. Emissary foramens of the human skull: Anatomical characteristics and its relations with clinical neurosurgery. Int. J. Morphol 31: 287-92.

Ginat DT, Ellika SK, Corrigan J. 2013. MultiDetector-Row Computed Tomography Imaging of Variant Skull Base Foramina. Journal of Computer Assisted Tomography 37: 481-85.

Gingsberg LE, Pruett SW, Chen MY, Elster AD. 1994. Skull base foramina of the middle cranial fossa: reassessment of normal variation with high resolution CT. Am. J. Neuroradiol 15: 28391.

Görürgöz, C, Paksoy CS. 2020. Morphology and morphometry of the foramen venosum: a radiographic study of CBCT images and literature review. Surgical and Radiologic Anatomy. Published Online https://doi.org/ 10.1007/s00276-020-02450-6.

Gupta N, Ray B, Ghosh S. 2005. Anatomic characteristics of foramen Vesalius. Kathmandu University Medical Journal 3: 55-58.

Kale A, Aksu F, Ozturk A, Gurses IA, Gayretli O, Zeybek FG, Bayraktar B, Ari ZOnder N. 2009. Foramen of Vesalius. Saudi Medical Journal 30: 56-59.

Kaplan M, Erol FS, Ozveren MF, Topsakal C, Sam B, Tekdemir I. 2007. Review of complications due to foramen ovale puncture. J. Clin. Neurosci 14: 563-68.

Kodama K, Inoue K, Nagashima M, Matsumura G, Watanabe S, Kodama G. 1997. Studies on the foramen Vesalius in the Japanese juvenile and adult skulls. Hokkaidolgaku Zasshi 72: 66774.

Lang J. 1983. Clinical anatomy of the head, neurocranium, orbit and craniocervical region. Springer, Berlin.

Lanzieri CF, Duchesneau PM, Rosenbloom $S A$, Smith AS, Rosenbaum AE. 1988. The Significance of Asymmetry of the Foramen of Vesalius. American Journal of Neuroradiology 9: 1201-04.

Lazarus L, Naidoo N, Satyapal KS. 2015. An osteometric evaluation of the foramen spinosum and venosum. Int. J. Morphol 33: 452-58.

Natsis K, Piagkou M, Repousi E, Tegos T, Gkioka A, Loukas M. 2018. The size of the foramen ovale regarding to the presence and absence of the emissary sphenoidal foramen: 
$¿$ is there any relationship between them? Folia Morphol 77: 90-98.

Ozer MA, Govsa F. 2014. Measurement accuracy of foramen of Vesalius for safe percutaneous techniques using computerassisted three-dimensional landmarks. Surgical and Radiologic Anatomy 36: 147-54.

Raval BB, Singh PR, Rajguru J. 2015. A morphologic and morphometric study of foramen Vesalius in dry adult human skulls of Gujarat region. Journal of Clinical and Diagnostic Research 9: 4-7.

Reymond J, Charuta A, Wysocki J. 2005. The morphology and morphometry of the foramina of the grater wing of the human sphenoid bone. Folia morphol 64: 188-93.

Rossi AC, Freire AR, Prado FB, Caria PHF, Botacin PR. 2010. Morphological characteristics of foramen of Vesalius and its relationship with clinical implications. Journal of Morphological Sciences 27: 26-29.
Rouviere H, Delmas A. 2005. Anatomía humana descriptiva, topográfica y funcional. Volumen 1. Edición 11. Barcelona. Editorial Masson. 544p.

Shinohara AL, de Souza Melo CG, Silveira EM, Lauris JR, Andreo JC, de Castro Rodrigues A. 2010. Incidence, morphology and morphometry of the foramen of Vesalius: complementary study for a safer planning and execution of the trigeminal rhizotomy technique. Surg Radiol Anat 32: 159-64.

Srimani $P$, Mukherjee $P$, Sarkar $M$, Roy $H$, Sengupta SK, Sarkar AN, Ray K. 2014. Foramina in alisphenoid - An observational study on their osseous-morphology and morphometry. Int. J. Anat. Radiol. Surg 3: 1-6

Ukoha U, Chijioke O, Chinwe U, Izuchukwu O, Nwankwo H, Ekezie J. 2018. Morphometric study of the jugular foramen in dry Nigerian skulls. Rev Arg de Anat Clin 10: 112-19. 